\title{
SARS-CoV-2-Impfungen - Was muss der Nephrologe wissen?
}

Autoren

Gunnar H. Heine ${ }^{1,2}$, Sören L. Becker ${ }^{3}$, Anja L. Scheuer ${ }^{1,2}$, Stephan H. Schirmer ${ }^{2,4}$

Institute

1 Medizinische Klinik II, AGAPLESION MARKUS KRANKENHAUS, Frankfurt

2 Universität des Saarlandes, Homburg

3 Institut für Medizinische Mikrobiologie und Hygiene, Universitätsklinikum des Saarlandes, Homburg

4 Kardiologische Praxis Schirmer, Kaiserslautern

Bibliografie

Dtsch Med Wochenschr 2021; 146: 466-470

DOI 10.1055/a-1375-4471

ISSN 0012-0472

(C) 2021. Thieme. All rights reserved.

Georg Thieme Verlag KG, Rüdigerstraße 14,

70469 Stuttgart, Germany
WAS IST NEU?

Impfstoffe Innerhalb von nur 15 Monaten nach Beginn der COVID-19-Pandemie stehen bereits mehrere SARS-CoV-2Impfstoffe zum klinischen Einsatz bereit. Während das Zielmolekül aller prädominierenden Vakzine das Spike-Protein von SARS-CoV-2 ist, lassen sie sich anhand ihres Wirkprinzip in mRNA-basierte, Vektor-basierte und Protein-basierte Impfstoffe unterscheiden.

Bedeutung für chronisch nierenkranke Menschen Das Aussprechen von Empfehlungen für chronisch nierenkranke Menschen ist aufgrund deren geringer Repräsentation in den Zulassungsstudien erschwert - wie bei anderen Patienten mit schwerer Komorbidität. Aufgrund von urämischem Immundefekt und/oder medikamentöser Immunsuppression könnte die Effektivität der Impfung im Einzelfall relativ vermindert sein. Dennoch profitieren vermutlich gerade diese Patienten von einer Impfung, da sie ein erhöhtes Risiko für einen schweren oder gar tödlichen COVID-19-Verlauf aufweisen.

\section{Stand der Dinge}

Als Antwort auf die von SARS-CoV-2 verursachte COVID-19Pandemie wurden in atemberaubender Geschwindigkeit innerhalb von weniger als 15 Monaten zahlreiche Impfstoffe entwickelt. Nachdem der erste Impfstoff noch im Jahr 2020 in Deutschland zugelassen wurde, sind zum Zeitpunkt der Manuskripterstellung (Ende Februar 2021) bereits 3 Impfstoffe zugelassen und für mindestens 2 weitere aussichtsreiche Kandidaten liegen vielversprechende Daten aus Phase-III-Studien vor.

Nahezu alle ärztlichen Kollegen werden daher in den ersten Monaten des Jahres 2021 im klinischen Alltag mit Fragen zu SARS-CoV-2-Impfstoffen konfrontiert, ohne dabei auf eigene Erfahrung oder traditionelle Hilfsmittel wie gedruckte Lehrbücher zurückgreifen zu können. Während sich in der Prävention von COVID-19 bei Gesunden oder Menschen mit leichten Komorbiditäten solche Fragen relativ stereotyp beantworten lassen und zahlreiche Informationshilfen von Fachgesellschaften und staatlichen Behörden zur Verfügung stehen, ergeben sich bei Patienten mit schwereren Komorbiditäten größere Herausforderungen bei der Indikationsstellung zur Impfung und bei der Auswahl einzelner Impfstoffe. Zu solchen komplex vorerkrankten Patienten gehören insbesondere auch Patienten mit chronischer Nierenerkrankung, da diese einerseits bei einer COVID-19-Erkrankung ein erhöhtes Risiko für einen schweren Krankheitsverlauf haben, andererseits aber aufgrund eines originären „urämischen Immundefektes“ und nicht selten aufgrund zusätzlicher Einnahme von immunsuppressiven Medikamenten möglicherweise ein vermindertes oder fehlendes Impfansprechen zeigen, wie dies bei chronisch nierenkranken Menschen etwa für Hepatitis- oder Influenzaimpfungen länger als ein Vierteljahrhundert bekannt ist [1].

Im folgenden Überblick sollen die Impfstoffe kurz charakterisiert werden, die Ende Februar 2021 in Deutschland bereits zugelassen sind oder deren Zulassung in den nächsten Monaten erwartet wird. Hierbei wird - soweit verfügbar - auf Ergebnisse von Phase-III-Studien fokussiert. Im Anschluss soll auf der Basis von Empfehlungen von Fachgesellschaften diskutiert werden, ob bei der Impfung von chronisch nierenkranken Menschen Besonderheiten zu beachten sind.

\section{Impfstoffe}

\section{mRNA-basierte Impfstoffe}

\section{BNT162b2}

In chronologischer Reihenfolge der Publikation der Phase-II/III-Studien waren zunächst die Daten des 
BNT162b2-mRNA-Impfstoffs der Firmen BioNTech und Pfizer verfügbar. Die in einem Lipid-Nanopartikel verpackte mRNA kodiert für eine leicht modifizierte Form des für das Andocken des Virus an die Wirtszelle wichtigen Spike-Proteins. In der Studie wurden 43448 Teilnehmer ( $\geq 16$ Jahre alt) randomisiert, entweder Placebo oder $30 \mu \mathrm{g}$ des Impfstoffs 2-mal im Abstand von 21 Tagen intramuskulär zu erhalten [2]. Der primäre Endpunkt der Studie war die durch Real-time-PCR nachgewiesene, symptomatische Infektion mit SARS-CoV-2, wobei Ereignisse ab 7 Tage nach der 2. Injektion gewertet wurden. Nach einer mittleren Nachbeobachtung von 2 Monaten trat der Endpunkt in 8 Fällen in der Impfstoffgruppe bzw. 162 Fällen in der Placebogruppe auf, entsprechend einer Effektivität von $95 \%$ (95\%-Konfidenzintervall (KI) 90,3$97,6 \%)$. Schwere Verläufe fanden sich bei 9 Patienten in der Placebogruppe, aber lediglich 1-mal in der Verumgruppe. Subgruppen für Alter, Geschlecht, Ethnizität oder Vorerkrankungen zeigten keine signifikanten bzw. relevanten Unterschiede in der Effektivität. Nebenwirkungen waren generell mild bis moderat, wobei eine lokale Reaktion an der Einstichstelle (am häufigsten leichte Schmerzen) in der Verumgruppe deutlich häufiger auftrat als unter Placebo. Generalisierte Nebenwirkungen (am häufigsten Abgeschlagenheit, Kopfschmerz, auch erhöhte Temperatur) fanden sich in der Verumgruppe zwar etwas häufiger, kamen aber auch in bis zu einem Drittel der Placebogruppe vor. Nebenwirkungen waren nach der 2. Impfung häufiger als nach der 1. und traten bei jüngeren Geimpften (<55 Jahre) stärker auf als bei älteren Personen.

mRNA-1273

In der COVE-Studie wurde der Impfstoff mRNA-1273 der Firma Moderna in einem ähnlichen Studiendesign wie der BNT162b2-mRNA-Impfstoff der Firmen BioNTech und Pfizer getestet. 30420 Menschen (>18 Jahre) wurden zu Placebo oder Impfstoff randomisiert, wobei in letzterer Gruppe $100 \mu \mathrm{g}$ des Impfstoffs im Abstand von 28 Tagen 2-mal intramuskulär injiziert wurden [3]. Symptomatische Infektionen wurden ab 14 Tage nach der 2. Injektion gewertet. Diese traten nach im Mittel 2 Monaten in der Placebogruppe 185-mal auf, in der Verumgruppe nur 11-mal, entsprechend einer Effizienz von 94,1\% (95\%-KI 89,3-96,8\%). Schwere Verläufe inklusive eines Todesfalls traten sämtlich in der Placebogruppe auf. Subgruppenanalysen zeigten keine relevanten Unterschiede, wobei ein Impfschutz bereits 14 Tage nach der ersten Infektion zu bestehen scheint (Effektivität 95,2\%; $95 \%$-KI 91,2$97,4 \%$ ) und auch Studienteilnehmer mit vorangegangener SARS-CoV-2-Infektion geschützt waren. Nebenwirkungen waren - ähnlich wie beim BNT162b2-Impfstoff häufig lokal an der Einstichstelle; systemische Impfreaktionen wie Müdigkeit, Kopfschmerzen oder Fieber waren meistens gering und etwas stärker ausgeprägt bei der Zweitinjektion.

\section{Vektor-basierte Impfstoffe}

\section{ChAdOx1 nCoV-19}

Der erste nicht-mRNA-basierte Impfstoff, der eine Marktzulassung in Deutschland erhielt, ist der AdenovirusImpfstoff der Firma AstraZeneca (ChAdOx1 nCoV-19, AZD1222), der ein replikationsdefizientes SchimpansenAdenovirus als Vektor benutzt, welches die DNA des SARS-CoV-2-Spike-Proteins trägt. Bisher publizierte Sicherheits- und Effektivitätsdaten basieren auf einer kumulativen Interimsanalyse von 4 randomisiert-kontrollierten Studien, in der Ergebnisse von 20675 Teilnehmern berichtet wurden [4]. 10-12\% der Teilnehmer waren älter als 55 Jahre. Als Endpunkt wurden symptomatische Infektionen mit positivem PCR-Ergebnis ab 14 Tage nach der 2. Dosis gewertet.

Die Beurteilung der Studien erschwert, dass die 4 Einzelstudien unterschiedliche Designs hatten und teilweise zunächst als „Single-Shot“-Studien angelegt waren. Erst im Verlauf wurden die betroffenen Probranden dann mit sehr unterschiedlich langer Latenz zur Zweitimpfung eingeladen, nachdem während des Studienverlaufs die Einfachimpfung nicht mehr als optimal betrachtet wurde. Zudem erhielten zahlreiche Probanden bei der Erstimpfung versehentlich eine deutlich niedrigere Impfstoffdosis als geplant.

Nach 2 Impfungen der geplanten $5 \times 10^{10}$ Viruspartikel in 4-wöchigem Abstand wird eine Effektivität von 62,1\% (95\%-KI 41,0-75,7\%) berichtet; wurde (versehentlich) nur die halbe Impfmenge bei der Erstinjektion verabreicht, fand sich eine Effektivität von $90 \%$ (95\%-KI 67,4$97 \%, 3$ von 1367 vs. 30 von 1174 Teilnehmern). Insgesamt errechnete sich hierdurch eine Effektivität von $70,4 \%$. Schwere Verläufe traten ausschließlich in der Kontrollgruppe auf. Schwerere Nebenwirkungen traten unter Placebo ähnlich häufig auf wie unter Verum.

Dieser Impfstoff wurde von der Europäischen Arzneimittelagentur (EMA) zur Anwendung bei Erwachsenen ohne Altersbeschränkung empfohlen. Die STIKO hat hiervon abweichend zunächst entschieden, den Impfstoff bei über 65-jährigen Menschen nicht zu empfehlen. Diese Entscheidung erfolgte nicht aufgrund von Hinweisen auf eine verminderte Effektivität, sondern allein aufgrund der geringen Anzahl von älteren Menschen im Studienkollektiv. Am 4. März 2021 teilte die STIKO auf der Homepage des Robert Koch-Instituts schriftlich mit, dass in einer aktualisierten Empfehlung keine obere Altersbegrenzung für diesen Impfstoff mehr vorhanden sein wird.

\section{Gam-COVID-Vac}

Auch der in Russland entwickelte Gam-COVID-Vac-Impfstoff („Sputnik V“) ist Adenovirus-basiert; er besteht aus 2 verschiedenen rekombinanten Vektoren vom Typ 26 
und 5 (rAd26 und rAd5) für die Erst- und Zweitimpfung (Abstand 21 Tage), wodurch verhindert werden soll, dass eine Immunaktivität gegen das Adenovirus auftritt und dessen Effektivität abschwächt [5]. 16501 Teilnehmer wurden in die Verum-, 5476 in die Placebogruppe randomisiert. 21 Tage nach der 1. Impfung (primärer Endpunkt) konnten PCR-bestätigte Infektionen mit 91,6\%iger Effektivität (95\%-KI 85,6-95,2\%) verhindert werden. Nebenwirkungen waren überwiegend leichtgradig; schwerere Nebenwirkungen traten in der Verumund Placebogruppe zu gleichen Teilen auf.

\section{Ad26.COV2.S}

Für den Adenovirus-basierten Impfstoff Ad26.COV2.S der Firma Johnson \& Johnson liegen aktuell nur begutachtet publizierte Ergebnisse von Phase-II-Studien vor. Bei 805 Teilnehmern wurde dieser in einem Einfach- bzw. Zweifach-Impfschema sowie in 2 Dosierungen $\left(5 \times 10^{10}\right.$ oder $1 \times 10^{11}$ ) getestet [6]. 29 Tage nach der 1 . Impfung zeigten sich neutralisierende Antikörpertiter bei $90 \%$ der Teilnehmer, nach 57 Tagen bei $100 \%$. Die 2. Impfung konnte den Titer um den Faktor 2,6-2,9 steigern, die gute Reaktion bereits nach einer Impfung könnte jedoch auch zu einer klinischen Effektivität durch nur Einmalgabe führen. Daher wurde neben einer konventionellen Phase-IIIStudie mit 2 Impfungen (ENSEMBLE 2, NCT 04614948) auch eine Phase-III-Studie mit einer Einmalgabe des Impfstoffs aufgelegt (ENSEMBLE, NCT 04505722). Zu letzterer liegen zum Zeitpunkt der Manuskripteinreichung erste (vor-) veröffentlichte Daten vor, die eine Reduktion von symptomatischen COVID-19-Erkrankungen von $66 \%$ und von schweren Erkrankungen von 85 \% aufweisen; die Effektivität gegen milde Erkrankung war in den USA und Lateinamerika größer als in Südafrika (72\%, 66 \% oder $57 \%)$. Insbesondere traten keine Todesfälle bei Geimpften auf. Eine Zulassung in den USA erfolgte Ende Februar 2021; die europäische Zulassung wurde zum Zeitpunkt des Redaktionsschlusses Ende März 2021 erwartet.

\section{Protein-basierte Impfstoffe}

Neben mRNA- und Vektor-basierten Impfstoffen gibt es auch im Bereich der klassischen sogenannten Totimpfstoffe oder inaktivierten Impfstoffe zahlreiche Initiativen zur Entwicklung von Impfstoffen gegen SARS-CoV-2. Hierbei werden entweder abgetötete Viren oder aber einzelne Proteine oder sogenannte virus-like particles (VLPS) eingesetzt, um eine Immunantwort hervorzurufen. Solche Impfstoffe benötigen häufig eine Kopplung mit einem Adjuvans, um eine ausreichend starke Impfantwort hervorzurufen, und sind im Herstellungsprozess aufwendiger, weshalb beispielsweise rasche Anpassungen des Impfstoffs beim Auftreten mutationshaltiger Virusvarianten weniger schnell möglich sind als z. B. bei mRNAbasierten Impfstoffen.
Zum Zeitpunkt der Manuskriptfertigstellung waren publizierte Phase-III-Daten zu diesen Impfstoffen noch nicht verfügbar. Diese werden u. a. von großen chinesischen Firmen (z. B. Sinovac, Sinopharm) sowie von verschiedenen europäischen (z. B. Sanofi, Valneva), amerikanischen (z. B. Novavax) und anderen Herstellern entwickelt.

\section{Vergleichende Wertung der COVID-19-Impfstoffe}

Auch wenn sich die Wirkprinzipien sowie die Gesamteffektivität der zugelassenen bzw. sich im Zulassungsprozess befindlichen Impfstoffe teilweise deutlich unterscheiden, so ist hervorzuheben, dass alle Impfstoffe sehr effektiv vor einem schweren Krankheitsverlauf, einer COVID-19-bedingten Hospitalisierung und einem COVID-19-assoziierten Tod schützen ( $\vee$ Tab. 1 ). Diese Parameter sind für den einzelnen Patienten von deutlich größerer Relevanz als die Gesamteffektivität, welche auch den Schutz vor asymptomatischen oder leichten Krankheitsverläufen beinhaltet, die aus klinischer Sicht jedoch häufig vernachlässigbar sind, da von ihnen nur ein geringes Risiko für den Patienten ausgeht. Wenn auch detaillierte Studiendaten zu einer reduzierten Transmission von SARS-CoV-2 durch Geimpfte noch nicht für alle Impfstoffe vorliegen, so ist von einer deutlichen Reduktion derselben auszugehen. Lokale oder systemische Impfreaktionen zeigen sich bei einem relativen großen Teil der Geimpften und sind häufiger als z. B. bei der Influenza-Impfung - bei mRNA-Impfstoffen kommt es eher nach der 2. Injektion zu solchen Reaktionen, wohingegen diese bei Vektor-basierten Impfstoffen nach der Erstgabe häufiger sind. Diese Impfreaktionen sind jedoch meist von kurzer Dauer (24-48 Stunden) und vorübergehend, schwere oder dauerhaft bleibende Schädigungen sind bisher nicht aufgetreten - es besteht jedoch ein geringes Risiko für anaphylaktische Reaktionen, sodass impfendes Personal entsprechend geschult sein sollte.

\section{Klinische Relevanz}

Obwohl leichte Unterschiede hinsichtlich des Schutzes vor milden COVID-19-Verläufen zu verzeichnen waren, verhinderten alle 5 bisher in Phase-III-Studien getesteten Vakzine zu nahezu $100 \%$ schwere und tödliche Verläufe von COVID-19, und außer zeitlich umschriebenen Impfreaktionen sind bislang keine relevanten Nebenwirkungen nachgewiesen.

\section{Übertragung der Studienergebnisse auf chronisch nierenkranke Menschen}

Nahezu alle bisher veröffentlichten Phase-I-III-Studien zu SARS-CoV-2-Impfstoffen fokussierten auf gesunde Probanden; zahlreiche Komorbiditäten und/oder die Einnahme von Immunsuppressiva waren als Ausschlusskriterium vordefiniert. Auch ältere Menschen sind in mehreren 
- Tab. 1 Vergleich der verschiedenen COVID-19-Impfstoffe.

\begin{tabular}{|c|c|c|c|c|c|}
\hline Vakzin & $\begin{array}{l}\text { BNT162b2 } \\
\text { BioNTech/Pfizer }\end{array}$ & $\begin{array}{l}\text { mRNA-1273 } \\
\text { Moderna }\end{array}$ & $\begin{array}{l}\text { ChAdOx1 nCoV-19 } \\
\text { AstraZeneca/Oxford }\end{array}$ & $\begin{array}{l}\text { Ad26.COV2.5 } \\
\text { Johnson \& Johnson }\end{array}$ & $\begin{array}{l}\text { Gam-COVID-Vac } \\
\text { („Sputnik V“) }\end{array}$ \\
\hline Wirkprinzip & mRNA & rRNA & Vektorviren & Vektorviren & Vektorviren \\
\hline Applikationsform & $\begin{array}{l}\text { 2-mal im Abstand } \\
\text { von } ~ 3 \text { Wochen }\end{array}$ & $\begin{array}{l}\text { 2-mal im } \\
\text { Abstand von } \\
\sim 4 \text { Wochen }\end{array}$ & $\begin{array}{l}\text { 2-mal im Abstand } \\
\text { von 9-12 Wochen }\end{array}$ & 1-mal & $\begin{array}{l}\text { 2-mal im } \\
\text { Abstand von } \\
3 \text { Wochen }\end{array}$ \\
\hline $\begin{array}{l}\text { Schutzwirkung gegen } \\
\text { jede Form von COVID }\end{array}$ & $95 \%$ & $94 \%$ & $\begin{array}{l}70 \% \text { (bei längerem } \\
\text { Dosisintervall bis } 90 \% \text { ) }\end{array}$ & $66-72 \%$ & $92 \%$ \\
\hline $\begin{array}{l}\text { Schutzwirkung gegen } \\
\text { schwere COVID-Verläufe }\end{array}$ & $>95 \%$ & $>95 \%$ & $>95 \%$ & $\sim 85 \%$ & $>95 \%$ \\
\hline $\begin{array}{l}\text { Schutz vor COVID-be- } \\
\text { dingtem Tod } 4 \text { Wochen } \\
\text { nach Impfung }\end{array}$ & 100\% & 100\% & 100\% & $\sim 100 \%$ & 100\% \\
\hline $\begin{array}{l}\text { Schutz gegen } \\
\text { Virusvarianten }\end{array}$ & $\begin{array}{l}\text { B.1.1.7 ja } \\
\text { B.1.351 etwas reduziert } \\
\text { P.1 ( } 501 Y . V .3 \text { ) noch } \\
\text { unklar }\end{array}$ & $\begin{array}{l}\text { B.1.1.7 ja } \\
\text { B.1.351 etwas } \\
\text { reduziert } \\
\text { P.1 (501Y.V.3) } \\
\text { noch unklar }\end{array}$ & $\begin{array}{l}\text { B.1.1.7 ja } \\
\text { B.1.351 reduziert (aber } \\
\text { wahrscheinlich Schutz vor } \\
\text { schweren Verläufen) } \\
\text { P.1 (501Y.V.3) noch unklar }\end{array}$ & $\begin{array}{l}\text { B.1.1.7 ja } \\
\text { B.1.351 und P.1 } \\
\text { (501Y.V.3) reduziert, } \\
\text { aber keine schweren } \\
\text { Verläufe }\end{array}$ & noch unklar \\
\hline
\end{tabular}

Phase-III-Studien unterrepräsentiert, insbesondere in der Zulassungsstudie für ChAdOx1 nCoV-19.

Daher müssen Nephrologen Studienergebnisse von der Allgemeinbevölkerung auf chronisch nierenkranke Menschen extrapolieren, bis spezifische Daten für nierenkranke Menschen vorliegen. Auch wenn die Impfantwort zumindest bei schwerer chronischer Nierenerkrankung und bei Einnahme von Immunsuppressiva eingeschränkt ist, gilt momentan als Expertenkonsensus, dass den meisten nephrologischen Patienten eine SARS-CoV-2-Impfung angeboten werden sollte. Selbst wenn die Effektivität der Impfung geringer als in der Gesamtbevölkerung ist (wofür es momentan noch keine solide Datenbasis gibt), könnte aufgrund des häufiger schweren Krankheitsverlaufs von COVID-19 bei chronisch nierenkranken Menschen der Benefit dennoch mindestens so bedeutsam sein wie in der Allgemeinbevölkerung. Verschiedene Fachgesellschaften haben Empfehlungen publiziert, die online verfügbar sind. Eine Präferenz für einen bestimmten Impfstofftyp gibt es nephrologischerseits aktuell weder aus Sicht der Autoren dieses Artikels, noch nach Meinung nationaler und internationaler Experten, auch wenn zuletzt Empfehlungen der Deutschen Transplantationsgesellschaft eine Präferenz für einzelne Substanzen andeuteten. Einen Sonderfall könnten frisch nierentransplantierte Patienten und Patienten mit B-Zell-depletierenden Medikamenten (z. B. Rituximab) sein, bei denen zur Gewährung einer ausreichenden Impfantwort eine Latenz zwischen maximaler Immunsuppression und Impfung erwägenswert erscheint. Umgekehrt gibt es aber bei diesen beiden Patientengruppen keinerlei Sicherheitsbedenken gegen eine Impfung, sodass manche
Experten eine sofortige Impfung auch unter laufender Therapie und eine Auffrischimpfung nach einigen Monaten favorisieren. Insbesondere bei der Diskussion um die Impfung unter Rituximab und die möglicherweise fehlende Antikörperbildung sollte beachtet werden, dass die Impfeffektivität neben humoralen auch zelluläre Komponenten umfasst, die von Rituximab weniger eingeschränkt werden.

\section{Klinische Relevanz}

Auch wenn die Effektivität der Impfung bei Patienten mit chronischer Nierenerkrankung vermindert sein könnte, profitieren sie aller Wahrscheinlichkeit nach dennoch von einer Impfung, da sie im Falle einer Infektion ein erhöhtes Risiko für einen schweren oder gar tödlichen COVID-19-Verlauf aufweisen.

\section{ZUSATZINFO}

Die Autoren dieses Beitrags haben weitere Informationen und Experteninterviews online zur Verfügung gestellt:

https://youtube.com/channel/UCFx_ZEsTtdpF PAyl5JVZ7kw.

\section{Interessenkonflikt}

Die Autorinnen/Autoren geben an, dass kein Interessenkonflikt besteht. 
Autorinnen/Autoren

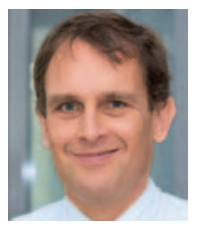

\section{Prof. Dr. med. Gunnar Heine}

Ist Chefarzt der Medizinischen Klinik II im Agaplesion Markus Krankenhaus, Frankfurt am Main, und APL-Professor an der Universität des Saarlandes.

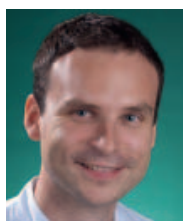

\section{Prof. Dr. Dr. Sören L. Becker}

ist Direktor des Instituts für Medizinische Mikrobiologie und Hygiene am Universitätsklinikum des Saarlandes. Er ist Facharzt für Medizinische Mikrobiologie, Virologie und Infektionsepidemiologie. Diploma in Tropical Medicine \& Hygiene (DTM\&H), Reisemedizin (DTG).

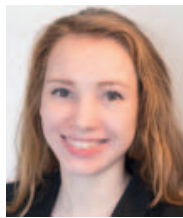

\section{Anja L. Scheuer}

ist Medizinstudentin im Praktischen Jahr am AGAPLESION MARKUS KRANKENHAUS und Mitglied der wissenschaftlichen Arbeitsgruppe „Kardiovaskuläre Erkrankungen bei chronisch nierenkranken Menschen“ der Universität des Saarlandes.

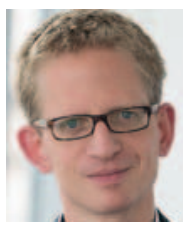

\section{Prof. Dr. Dr. Stephan Schirmer}

ist Leiter der Kardiopraxis Schirmer als Internist, Kardiologe und Angiologe sowie apl.-Professor an der Universität des Saarlandes.
Korrespondenzadresse

Prof. Dr. med. Gunnar Henrik Heine

Agaplesion Markus Krankenhaus

Medizinische Klinik II

Wilhelm-Epstein-Straße 4

60431 Frankfurt am Main

Deutschland

gunnar.heine@fdk.info

Literatur

[1] Kohler $\mathrm{H}$, Girndt M, Dumann $\mathrm{H}$ et al. Immune defect in renal failure (Part I). Dtsch Med Wochenschr 1993; 118: 757-761. doi:10.1055/s-2007-1024155

[2] Polack FP, Thomas S], Kitchin $\mathrm{N}$ et al. Safety and Efficacy of the BNT162b2 mRNA Covid-19 Vaccine. N Engl J Med 2020; 383 : 2603-2615. doi:10.1056/nejmoa2034577

[3] Baden LR, El Sahly HM, Essink B et al. Efficacy and Safety of the mRNA-1273 SARS-CoV-2 Vaccine. N Engl J Med 2021; 384: 403-416. doi:10.1056/nejmoa2035389

[4] Voysey M, Clemens SAC, Madhi SA et al. Safety and efficacy of the ChAdOx1 nCoV-19 vaccine (AZD1222) against SARS-CoV-2: an interim analysis of four randomised controlled trials in Brazil, South Africa, and the UK. Lancet 2021; 397: 99-111. doi:10.1016/S0140-6736(20)32661-1

[5] Logunov DY, Dolzhikova IV, Shcheblyakov DV et al. Safety and efficacy of an rAd26 and rAd5 vector-based heterologous prime-boost COVID-19 vaccine: an interim analysis of a randomised controlled phase 3 trial in Russia. Lancet 2021; 397: 671681. doi:10.1016/S0140-6736(21)00234-8

[6] Sadoff ], Le Gars M, Shukarev G et al. Interim Results of a Phase 1-2a Trial of Ad26.COV2.S Covid-19 Vaccine. N Engl J Med 2021. doi:10.1056/nejmoa2034201 